\title{
Hasselhorn, Benjamin
}

\section{Erinnerung im Streit. Zum Umgang der deutschen Geschichtswissenschaft mit ihrer eigenen Vergangenheit}

Erziehungswissenschaft 25 (2014) 49, S. 27-36

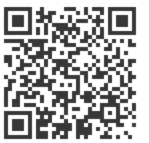

Quellenangabe/ Reference:

Hasselhorn, Benjamin: Erinnerung im Streit. Zum Umgang der deutschen Geschichtswissenschaft mit ihrer eigenen Vergangenheit - In: Erziehungswissenschaft 25 (2014) 49, S. 27-36 - URN:

urn:nbn:de:0111-pedocs-100771 - DOI: 10.25656/01:10077

https://nbn-resolving.org/urn:nbn:de:0111-pedocs-100771

https://doi.org/10.25656/01:10077

in Kooperation mit / in cooperation with:

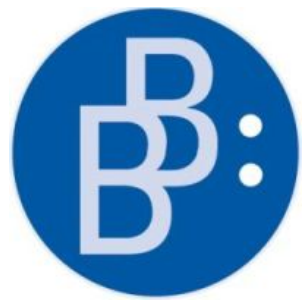

https://www.budrich.de

\section{Nutzungsbedingungen}

Gewährt wird ein nicht exklusives, nicht übertragbares, persönliches und beschränktes Recht auf Nutzung dieses Dokuments. Dieses Dokument ist ausschließlich für den persönlichen, nicht-kommerziellen Gebrauch bestimmt. Die Nutzung stellt keine Übertragung des Eigentumsrechts an diesem Dokument dar und gilt vorbehaltlich der folgenden Einschränkungen: Auf sämtlichen Kopien dieses Dokuments müssen alle Urheberrechtshinweise und sonstigen Hinweise auf gesetzlichen Schutz beibehalten werden. Sie dürfen dieses Dokument nicht in irgendeiner Weise abändern, noch dürfen Sie dieses Dokument für öffentliche oder kommerzielle Zwecke vervielfältigen, öffentlich ausstellen, aufführen, vertreiben oder anderweitig nutzen.

Mit der Verwendung dieses Dokuments erkennen Sie die Nutzungsbedingungen an.

\section{Terms of use}

We grant a non-exclusive, non-transferable, individual and limited right to using this document.

This document is solely intended for your personal, non-commercial use. Use of this document does not include any transfer of property rights and it is conditional to the following limitations: All of the copies of this documents must retain all copyright information and other information regarding legal protection. You are not allowed to alter this document in any way, to copy it for public or commercial purposes, to exhibit the document in public, to perform, distribute or otherwise use the document in public.

By using this particular document, you accept the above-stated conditions of use.

\section{Kontakt / Contact:}

\section{peDOCS}

DIPF | Leibniz-Institut für Bildungsforschung und Bildungsinformation Informationszentrum (IZ) Bildung

E-Mail: pedocs@dipf.de

Internet: www.pedocs.de

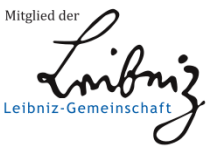




\section{Erziehungswissenschaft}

Mitteilungen der Deutschen Gesellschaft

für Erziehungswissenschaft (DGfE)

Heft 49

25. Jahrgang 2014

ISSN 0938-5363

Verlag Barbara Budrich 


\section{INHALTSVERZEICHNIS}

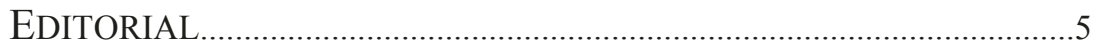

BEITRÄGE ZUM THEMA ,ERINNERUNGSKULTUREN PREISE, NAMEN UND DISZIPLINPOLITIK“

Stellungnahme des Vorstandes zur Rücknahme des Namens für den

Forschungspreis vom 13. Januar 2014

Andreas Hoffmann-Ocon

Heinrich Roth und der Preis der Forschung - Historisierungen,

Verundeutlichungen und erziehungswissenschaftliche

Erinnerungsgeschichten

Benjamin Hasselhorn

Erinnerung im Streit. Zum Umgang der deutschen

Geschichtswissenschaft mit ihrer eigenen Vergangenheit

Eva Matthes \& Carola Groppe

Historische Bildungsforschung und Erinnerungskultur. Eine

Stellungnahme der Sektion Historische Bildungsforschung.

Daniel Tröhler

Wissenschaftliche Ehrungen oder der doppelte Gestus der

Auszeichnung. Bemerkungen zur fallengelassenen Idee eines Heinrich

Roth-Forschungspreises der DGfE

Jörg Schlömerkemper

„Nomen est omen“ - oder geht es auch ohne? Was kann/Was soll der

Name einer Person bei einem Preis bedeuten? Anmerkungen nach dem

Streit um die Benennung des Forschungspreises der DGfE nach

Heinrich Roth

BEITRÄGE

Adolf Kell

Grenzgänge, Traditionen und Zukünfte in der Deutschen Gesellschaft für Erziehungswissenschaft. Kongresse zur Reflexion - auch für die Sektion Berufs- und Wirtschaftspädagogik? 


\section{MitTEILUNGEN DES VORSTANDS}

Protokoll der Mitgliederversammlung der Deutschen Gesellschaft für

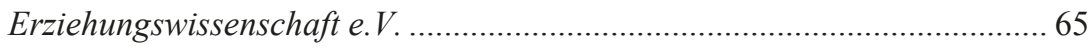

Ergebnis der Wahl zum Vorsitz und Vorstand der DGfE............................ 71

Liste der unbekannt verzogenen Mitglieder ............................................... 72

BERICHTE AUS DEN SEKTIONEN

Sektion 1 - Historische Bildungsforschung ............................................... 75

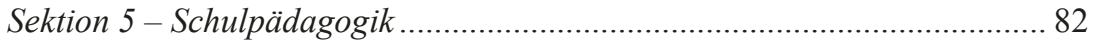

Sektion 8 - Sozialpädagogik und Pädagogik der frühen Kindheit................ 85

Sektion 9 - Erwachsenenbildung .............................................................. 91

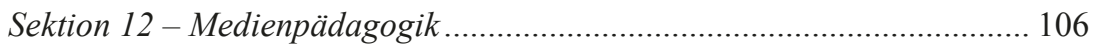

Sektion 13 - Differentielle Erziehungs- und Bildungsforschung ................ 108

NOTIZEN

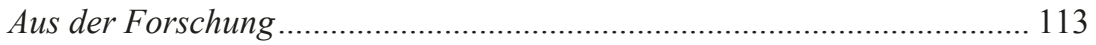

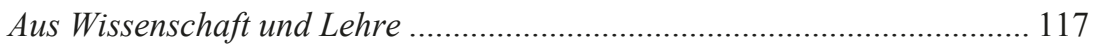

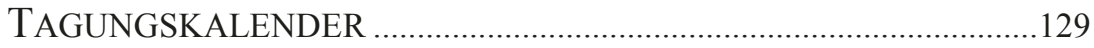




\title{
Erinnerung im Streit
}

\section{Zum Umgang der deutschen Geschichtswissenschaft mit ihrer eigenen Vergangenheit}

\author{
Benjamin Hasselhorn
}

Die Beziehungen deutscher Historiker zum nationalsozialistischen Regime sowie das Verhältnis der deutschen Geschichtswissenschaft der ersten Hälfte des 20. Jahrhunderts zum Nationalsozialismus insgesamt sind nach wie vor äußerst kontrovers diskutierte Streitthemen in der Disziplin und immer mal wieder auch im Historikerverband. Zum Ausdruck kam dies jüngst wieder anlässlich der von Christoph Nonn verfassten Biographie des Kölner Historikers Theodor Schieder (1908-1984), einer der bedeutendsten Vertreters der historischen Zunft in der Nachkriegszeit. In der Nonnschen Biographie wird die Entwicklung von Schieders politischem Denken rekonstruiert, der vor 1933 konservativ gewesen sei, sich nach 1933 zunächst angepasst, dann sogar weitgehend mit dem Nationalsozialismus identifiziert habe und der nach 1945 zwar ins bürgerlich-konservative Lager zurückgekehrt sei, eine „Aufarbeitung" der eigenen Vergangenheit aber unterlassen habe (Nonn 2013). Gegen diese Darstellung hat einer der „Pioniere“ (Nonn 2014) der „Aufarbeitung" der Geschichtswissenschaft im Nationalsozialismus, Peter Schöttler, massive Einwände erhoben: Nonn spiele die nationalsozialistische Verstrickung Schieders systematisch herunter, verwische die Kontinuität seines rechtsradikalen Denkens vor, während und letztlich auch nach der nationalsozialistischen Herrschaft, teile Schieders „Realitätsverweigerung“ in Bezug auf die Deutung von 1968 als einer 1933 strukturell ähnlichen historischen Situation und leugne das Netzwerk von Altnazis, das Schieder 1947 den Lehrstuhl in Köln verschafft habe (Schöttler 2013).

Diese neue Debatte um den ,Fall' Schieder ist ein Beleg für eine Problematik, die wiederum Schöttler selbst bei anderer Gelegenheit explizit benannt hat:

„Doch tatsächlich verwandeln sich fast alle zeitgeschichtlichen Sachdiskussionen noch immer - oder mehr denn je? - sehr schnell in Kontroversen oder polemische Konfrontationen. Meist fängt es schon bei der Sprache an, bei der Wortwahl: So als ob auch nach fast 60 Jahren nicht nur nichts vergessen wäre - zumindest expressis verbis wünscht das ja niemand -, sondern jede Positionierung in diesem Fragengeflecht noch immer politische Bedeutung hätte. Es wird abzuwarten sein, ob Historiker über ihre eigene Disziplin in Zukunft diskutieren können, ohne sich ständig, sobald es brisant wird, entweder der Übertreibung oder der Untertreibung zu verdächtigen." (Schöttler 2007: 15f.) 
Dementsprechend stand und steht die Ausbildung einer gemeinsamen Erinnerungskultur des Faches in Bezug auf die eigene jüngere Vergangenheit vor einer ganzen Reihe von Schwierigkeiten. Das begann schon in der unmittelbaren Nachkriegszeit, als man zwar einerseits die politisch eindeutig ,Belasteten" aus den Universitäten entfernte und sich durchaus einer selbstkritischen Bestandsaufnahme der eigenen Geschichte - und auch der eigenen Geschichtsschreibung - widmete, andererseits aber keinen Anlass zu einer Totalrevision sah und vor allem die zahlreichen „Angepassten“ und „Mitläufer“ unter den Kollegen schonte (vgl. Schulze 1997: $257 \mathrm{ff}$. und 342ff.). ${ }^{1}$ Das war sicherlich auch dem Umstand geschuldet, dass die zunächst mit erheblichem Aufwand vor allem von Seiten der US-Militärregierung eingeleitete „Entnazifizierung“ mit dem Ziel einer „Umerziehung“ einen gewissen Gegenreflex auslöste, der die verbliebenen Vertreter der deutschen Geschichtswissenschaft in eine Verteidigungshaltung geraten und dadurch näher zusammenrücken ließ (vgl. Schulze/Helm/Ott 1999: 11ff). Im Hinblick auf das Verhalten der deutschen Historiker während der nationalsozialistischen Herrschaft setzte sich daher relativ rasch als kanonische Deutung die Auffassung durch, dass abgesehen von einigen ,wildgewordene[n] Studienräte[n] und Außenseiter[n]" die deutsche Geschichtswissenschaft gebührende Distanz gegenüber der Ideologie des Regimes gehalten habe (Rothfels 1965: 90ff; vgl. außerdem Schulze 1989: 46ff.)

Dieser Konsens geriet erst im Laufe der 1960er Jahre ins Wanken, als in einigen Arbeiten die Haltung der deutschen Historiker zwischen 1933 und 1945 gegenüber dem Nationalsozialismus als mehrheitlich positiv geschildert wurde: Zwar habe man gewisse Vorbehalte gegenüber den Methoden des Regimes gehabt, sei aber mit dessen wesentlichen politischen Zielen, in erster Linie der Revision der Bestimmungen des Versailler Vertrags, ganz einverstanden gewesen. Nur in Bezug auf die eigene geschichtswissenschaftliche Arbeit selbst habe man sich gegen nationalsozialistische Einflussnahmen mehrheitlich zur Wehr gesetzt (so vor allem Werner 1967: bes. 61ff.; siehe außerdem Heiber 1966). Für derartige Differenzierungen war allerdings schon Ende der 1960er Jahre kaum mehr Platz angesichts eines politischen Klimas, in dem die „Aufarbeitung“ der nationalsozialistischen Vergangenheit nicht nur massiv gefordert und vorangetrieben wurde, sondern sich auch als politische Waffe in einem aufziehenden Generationenkampf erwies. Dies wiederum führte dazu, dass die angegriffenen Ordinarien sich jeder offenen Diskussion verweigerten und Fragen zu ihrem eigenen Verhalten in der NSZeit nach Möglichkeit auswichen. phal und Gustav Adolf Rein versagt, ohne dass dies allerdings ihre Möglichkeiten publizistischer Einflussnahme beschnitt. Eine selbstkritische Bestandsaufnahme findet sich etwa bei Friedrich Meinecke (1946/1969), aber auch bei Gerhard Ritter (1947). 
Spätestens in dieser Phase kam eine weitere Problematik hinzu, die die geschichtswissenschaftliche Beschäftigung mit der eigenen Disziplin erschwerte: die direkten persönlichen Beziehungen zwischen Forscher und Forschungsgegenstand. Gerade in den Reihen der als besonders ,kritisch' geltenden „Historischen Sozialwissenschaft“ Bielefelder Provenienz befanden sich Historiker, deren akademische Lehrer für die Jahre zwischen 1933 und 1945 alles andere als eine, weiße Weste' vorzuweisen hatten. ${ }^{2}$ Aufgrund der persönlichen Bindungen, auch der Dankbarkeit für die Unterstützung der eigenen Karriere scheuten sich aber gerade diejenigen, die permanent nach Kontinuitäten in der deutschen Geschichte auf ihrem angeblichen ,Sonderweg' in den Nationalsozialismus suchten, die wirklichen oder vermeintlichen Verstrickungen des eigenen Faches offen zu thematisieren. ${ }^{3}$

Erschwert wurde die Situation zusätzlich durch den Umstand, dass gerade die ,innovativen' Historiker, die schon früh sozialgeschichtlich gearbeitet hatten, als ,belastet' gelten mussten, während umgekehrt zahlreiche Vertreter traditioneller historiographischer Methoden nachweislich , unbelastet' waren. Und auch dort, wo der Gegensatz kein methodischer, sondern ein inhaltlicher oder sogar handfest politischer war wie etwa in der so genannten „FischerKontroverse" über den deutschen Schuldanteil am Ausbruch des Ersten Weltkriegs, stand auf Seiten der ,Progressiven' mit Fritz Fischer ein ehemaliger NS-Sympathisant, auf Seiten der ,Konservativen“ dagegen mit Gerhard Ritter ein NS-Gegner. ${ }^{4}$

Die Fachgesellschaft, der Verband der Historiker und Historikerinnen Deutschlands, bot Ende der 1990er dem Thema und seiner Problematik eine öffentliche Bühne: Auf dem vom Historikerverband veranstalteten 42. Deutschen Historikertag 1998 in Frankfurt am Main wurde eine vielbeachtete Sektion über „Historiker im Nationalsozialismus“ durchgeführt, in der sich die Schwierigkeiten einer zeitgeschichtlichen Erforschung des eigenen Faches noch einmal bündelten. Die vornehmlich jüngeren Historiker, die die

2 Das gilt insbesondere für Hans-Ulrich Wehler und - allerdings nicht vorbehaltlos der „Bielefelder Schule“ zuzuordnen - Wolfgang J. Mommsen, beide Schüler Theodor Schieders, außerdem für Jürgen Kocka, zu dessen akademischen Lehrern auch Werner Conze gehörte.

3 Zur von Wehler vertretenen Sonderwegthese vgl. Wehler 1983: bes. 11. Dort nennt Wehler als sein Hauptanliegen die Suche nach den Bedingungen dafür, dass Deutschland sich bis zur Mitte des 20. Jahrhunderts nicht ,zu einer Gesellschaft mündiger, verantwortlicher Staatsbürger“" entwickelt habe, sondern ,in die Katastrophe des deutschen Faschismus“ geraten sei. Als Hauptbedingungen werden dann die sozioökonomische Rückständigkeit Deutschlands im Vergleich zu Westeuropa sowie die verspätete Nationalstaatsbildung genannt. Diese Argumentation ist schon früh widerlegt worden, vor allem von Blackbourn/ Eley 1980.

4 Zu Fischers Parteinahme für den Nationalsozialismus und die Glaubensbewegung „Deutsche Christen“ zwischen 1933 und 1945 vgl. Große Kracht 2003. Fischer wiederum äußerte im Zuge der Kontroverse Zweifel an der demokratischen Gesinnung Ritters (vgl. Fischer 1977: 13 und 246f.). 
Sektion durchführten, erhoben zum Teil schwere Vorwürfe gegen führende Vertreter ihrer Zunft: Diese hätten nicht nur durch Passivität, sondern auch durch aktive Mitarbeit ein erhebliches Maß an Mitschuld an den nationalsozialistischen Verbrechen auf sich geladen (vgl. dazu vor allem Aly 1999: 163ff.). Historiker der älteren Generation wie Wolfgang J. Mommsen und Hans-Ulrich Wehler protestierten dagegen und warben um Gerechtigkeit für ihre akademischen Lehrer. Vor allem Wehler, der noch im 1986 begonnenen „Historikerstreit" unmissverständlich Partei ergriffen hatte gegen jeden Versuch einer „Historisierung ${ }^{65}$ des Nationalsozialismus, trat als Verteidiger seines Lehrers Theodor Schieder auf, dem er zwar keine ausdrückliche Aufarbeitung der eigenen Vergangenheit bescheinigte, aber doch einen ,politischreflexiven Lernprozeß“, der sich vor allem in einer vorsichtigen Distanzierung von einer nationalen Perspektive der Geschichtsschreibung und der Offenheit für methodische und theoretische Innovationen geäußert habe (Wehler 1999: 333).

Eine gemeinsame und identifizierbare Erinnerungskultur der Geschichtswissenschaft konnte sich unter diesen Umständen kaum ausbilden; eher wird man von einer ausgesprochenen Konfliktkultur in der Frage der Erinnerung und Beurteilung der eigenen Fachgeschichte sprechen müssen. Die verschiedenen Fachgesellschaften vertraten dabei je nach ihrer personellen Zusammensetzung mitunter divergierende Interessen, was sich wiederum am Umgang mit Theodor Schieder zeigen lässt: 1985 führte das Historische Kolleg, eine Gründung des Stifterverbandes für die Deutsche Wissenschaft, der Deutschen Bank und der Historischen Kommission bei der Bayerischen Akademie der Wissenschaften, deren Präsident Schieder bis zu seinem Tod 1984 gewesen war, eine „Theodor-Schieder-Gedächtnisvorlesung“ ein, die allerdings 2002 wieder umbenannt wurde, offenbar unter dem Eindruck der durch den Historikertag von 1998 initiierten Debatte (vgl. Schöttler 2013).

Es ist vor diesem Hintergrund wenig verwunderlich, dass sich das Ineinander von wissenschaftlichen, politischen und rein persönlichen Motiven bei Stellungnahmen zu diesem Thema nicht nur in dem wenig einheitlichen erinnerungskulturellen Umgang mit der Geschichte der Geschichtswissenschaft im Nationalsozialismus niederschlägt, sondern auch in der Forschungsdiskussion selbst. Dabei besteht durchaus ein Konsens darüber, dass die deutsche Geschichtswissenschaft sich im Großen und Ganzen als weitgehend „resistent" gegenüber dem Nationalsozialismus erwiesen habe. ${ }^{6}$ Gemeint ist damit der belegbare Sachverhalt, dass der Großteil der deutschen Historiker sich mehr oder weniger offen gegen jeden Versuch von Seiten des NS-Regimes

Den Begriff in die Debatte eingeführt hat Martin Broszat (1985). Zum Historikerstreit aus Sicht Wehlers vgl. Wehler 2006.

6 Klassisch dazu: Werner 1967:. 61. Neuer und unter Nennung der wichtigsten Forschungsliteratur: Wolf 1996: $15 \mathrm{ff}$. und S. 389ff.. 
wehrte, im Namen einer „deutschen“ oder „kämpfenden Wissenschaft“ (Frank:1934; vgl. dazu auch Heiber 1966). bis dahin gültige Regeln methodisch sauberer Wissenschaftlichkeit außer Kraft zu setzen. Gerade bei denjenigen konservativen Historikern, die mit der nationalsozialistischen „Machtergreifung" anfänglich gewisse politische Hoffnungen verknüpften, ist eine seit 1933 immer deutlicher werdende Gereiztheit zu beobachten angesichts tatsächlicher oder auch nur befürchteter Versuche des Regimes, Einfluss auf die eigene wissenschaftliche Arbeit zu nehmen. Ein Vortrag wie der des damals bereits emeritierten Tübinger Historikers Johannes Haller über die „Aufgaben des Historikers“, der ein an Leopold von Ranke orientiertes Objektivitätsideal vertrat und dieses gegen die nationalsozialistische ,mythische Schau der Vergangenheit" profilierte, welche auf reine subjektive "Willkür" hinauslaufe und „Spott mit der Wissenschaft“ treibe, löste bei seiner Publikation 1935 in der Zunft allgemeine Zustimmung aus (Haller 1935: 4 und 28ff.). ${ }^{7}$ Selbst Parteimitglieder wie Hallers Schüler und Tübinger Nachfolger Heinrich Dannenbauer brachen mit der Wissenschaftspolitik der NSDAP und bekannten sich offen zur „Voraussetzungslosigkeit“" der Wissenschaft. ${ }^{8}$

Strittig ist allerdings die Frage, wie dieser Sachverhalt der "Resistenz“ der deutschen Historiker auf ihrem eigenen Fachgebiet gegenüber dem Nationalsozialismus politisch zu beurteilen ist. Während die einen auf die faktische Unversehrtheit des Faches verweisen und die dadurch bewirkte „Störung“ (Werner 1967: 68). des NS-Regimes positiv würdigen, werfen die anderen den deutschen Historikern vor, gerade durch ihren, einem falschen Objektivitätsideal huldigenden, „Historismus“ dem Regime einen Dienst erwiesen zu haben und verweisen in diesem Zusammenhang auf die "Brauchbarkeit" (Schönwälder 1992: 15ff. und 274) der methodisch korrekt arbeitenden, inhaltlich aber aufgrund ihres Konservatismus NS-nahen Historiker für die Legitimation der nationalsozialistischen Herrschaft. Das Beharren der deutschen Geschichtswissenschaft auf den eingeübten wissenschaftlichen Standards sei vor allem deshalb so wenig beeindruckend, weil in den normativen Urteilen sowieso eine weitgehende Kongruenz zu den politischen Vorstellungen der NS-Führung bestanden habe (ebd.: 268).

Die Streitfrage über die deutschen Historiker im Nationalsozialismus erweist sich daher bei näherer Betrachtung als eine Streitfrage erstens über konservative Historiker im Nationalsozialismus im Hinblick auf die Frage,

Vgl. außerdem die Zusammenstellung von Rezensionen zu Hallers Aufsatz in: Universitätsarchiv Tübingen, Nachlass Johannes Haller 305/6; außerdem Real 1997: 96.

8 „Ich muß das heute, wo ich mit der herkömmlichen akademischen Feierlichkeit von meinem Lehrstuhl Besitz ergreife, ganz deutlich aussprechen: ich bekenne mich zu dem Grundsatz der voraussetzungslosen Forschung. [...] Nur beschränkte Geister können glauben, dem Staat zu nützen, indem sie dem Forscher vorschreiben, welche Ansicht er in bestimmten wissenschaftlichen Fragen, die sie selbst meist nur ungenügend kennen, vorzutragen habe.“(Dannenbauer 1935: 17ff.) 
ob Konservatismus und Nationalsozialismus in einem Harmonie- oder in einem Spannungsverhältnis zueinander stehen; zweitens über die Beurteilung der Legitimität oder Nichtlegitimität einer Beziehung zum nationalsozialistischen Regime, die nicht zu aktivem politischem Widerstand führte. Was die erste Frage betrifft, so verweisen Vertreter der Harmoniethese auf prinzipielle Gemeinsamkeiten zwischen Konservatismus und Nationalsozialismus wie etwa die Hochschätzung der Nation oder die „Machtstaatstheorie“ (Schleier 1975: bes. 61; Werner 1967: 69 und 88f.; Schönwälder 1992: 270ff.). Allerdings finden sich diese ideologischen Elemente auch in anderen politischen Lagern als dem Konservatismus, während umgekehrt der Nationalsozialismus keineswegs auf genau diese Elemente reduziert werden kann. Dessen Ideologeme - darunter auch die Rassenlehre - haben sich vielmehr seit dem 18. Jahrhundert herausgebildet und stehen ,,mit den Grundmotiven ihrer radikalen Traditionsfeindschaft, ihrer konsequenten Zukunftsorientierung, ihrem genuinen Antiindividualismus und Antiliberalismus" (Kraus 2011: 39) konservativen Vorstellungen - die wiederum alles andere als ideologisch einheitlich sind - eher entgegen. Dass die deutsche Geschichtswissenschaft bis 1945 - und auch noch darüber hinaus - mehrheitlich politisch konservativ war, ist daher kaum als Kriterium zur Diskreditierung geeignet.

Die zweite Frage dagegen ist kaum eindeutig zu beantworten, schon gar nicht mit den Mitteln der Geschichtswissenschaft. Dieser hat man jenseits totalitärer Wissenschaftskonzepte normalerweise nicht die Aufgabe zugewiesen, aus der Perspektive der Nachgeborenen über die Verhaltensweisen der eigenen Vorgänger Gericht zu halten, sondern hat vielmehr von ihr erwartet, vergangene Handlungsspielräume auszuloten und die dokumentierten und belegten Verhaltensweisen verständlich zu machen. Im Hinblick auf die NSZeit ist dabei bereits einige Differenzierungsarbeit geleistet und zutreffend festgestellt worden, dass es nicht nur um „Widerstand“ oder „Mitmachen“ ging, sondern dass es dazwischen noch sehr viel Raum für unterschiedliche Grade ,innerer Emigration“ oder aber „Anpassung“" gab.

Die Notwendigkeit, sich auf ,Zwischentöne“ (Cornelißen 2001: 229) einzulassen, wird dabei in erster Linie von einer neuen Historikergeneration betont. ${ }^{9}$ Das gilt auch für die eingangs erwähnte Kontroverse zwischen Schöttler und Nonn, in der letzterer sich gegen ein Selbstverständnis der Geschichtswissenschaft als einer Art Staatsanwaltschaft verwehrt und den an

In den letzten Jahren ist eine ganze Reihe von Historiker-Biographien erschienen, die in Bezug auf die Beziehungen der deutschen Historiker zum Nationalsozialismus auf Differenzierung Wert legen: Nonn 2013; Reichert: 2009; Thimme 2006; Eckel 2005. Der Verfasser dieses Aufsatzes bereitet eine biographische Studie über Johannes Haller vor. Bereits erschienen ist: Hasselhorn (Bearb.)/Kleinert (Vorarb.)2014. Die Geschichte des Historikerverbandes findet ebenfalls erst seit kurzem verstärkte Aufmerksamkeit (vgl. Cornelißen/ Sauer/Schulze 2013). 
ihn herangetragenen Vorwürfen entgegengehalten hat, es gehe bei der Geschichtsschreibung, ,nicht allein darum, ob etwas gut oder schlecht war", sondern darum, „warum es so gekommen ist, wie es kam“ (Nonn 2014).

Dahinter steht die Überzeugung, dass Sachanalyse und normative Beurteilung beide profitieren, wenn man sie voneinander trennt, und dass ein solches Vorgehen immer noch besser ist, als eines, in dem der Wunsch, eindeutig Position zu beziehen und die eigenen Erkenntnisse dadurch vor unliebsamer Deutung und Nutzung zu schützen, beides untrennbar miteinander vermischt. Wenn sich diese Auffassung im Fach allgemein durchsetzen würde, wäre dies ein Schritt in Richtung einer sachlicheren wissenschaftlichen Diskussion. Ob es auch ein Schritt in Richtung einer gemeinsamen Erinnerungskultur wäre, ist allerdings fraglich: Der (wissenschafts-)politische Umgang mit der Vergangenheit der eigenen Disziplin und seiner Vertreter wird durch eine weniger emotional aufgeladene Diskussionskultur möglicherweise entspannter, dürfte aber doch grundsätzlich umstritten bleiben. Denn hierbei handelt es sich nicht um wissenschaftliche, sondern um politische Entscheidungen.

Benjamin Hasselhorn, Dr., ist wissenschaftlicher Mitarbeiter für die Stiftung Luthergedenkstätten in Sachsen-Anhalt, Lutherstadt Wittenberg.

\section{Literatur}

Aly, Götz (1999): Theodor Schieder, Werner Conze oder Die Vorstufen der physischen Vernichtung. In: Schulze, W./Oexle, O. G: (Hrsg.): Deutsche Historiker im Nationalsozialismus. Frankfurt am Main: Fischer Taschenbuch Verlag, S. 163-182.

Blackbourn, David/Eley, Geoff (1980): Mythen deutscher Geschichtsschreibung. Die gescheiterte bürgerliche Revolution von 1848. Frankfurt am Main u.a.: Ullstein.

Broszat, Martin (1985): Plädoyer für eine Historisierung des Nationalsozialismus. In: Merkur 39, 435, S. 373-385.

Cornelißen, Christoph (2001): Gerhard Ritter. Geschichtswissenschaft und Politik im 20. Jahrhundert (= Schriften des Bundesarchivs 58). Düsseldorf: Droste.

Cornelißen, Christoph/Sauer, Michael/Schulze, Winfried (Hrsg.) (2013): Editorial. In: Geschichte in Wissenschaft und Unterricht 64, S. 3-4.

Dannenbauer, Heinrich (1935): Germanisches Altertum und deutsche Geschichtswissenschaft (= Philosophie und Geschichte 52). Tübingen: Mohr Siebeck.

Eckel, Jan (2005): Hans Rothfels. Eine intellektuelle Biographie im 20. Jahrhundert (Moderne Zeit. Neue Forschungen zur Gesellschafts- und Kul- 
turgeschichte des 19. und 20. Jahrhunderts 10). Göttingen: Wallstein Verlag.

Fischer, Fritz (1977): Der Erste Weltkrieg und das deutsche Geschichtsbild. Beiträge zur Bewältigung eines historischen Tabus. Aufsätze und Vorträge aus drei Jahrzehnten. Düsseldorf: Droste.

Frank, Walter (1934): Kämpfende Wissenschaft. Mit einer Vorrede des Reichsjugendführers Baldur von Schirach. Hamburg: Hanseatische Verlagsanstalt.

Große Kracht, Klaus (2003): Fritz Fischer und der deutsche Protestantismus. In: Zeitschrift für Neuere Theologiegeschichte 10, S. 224-252.

Haller, Johannes (1935): Über die Aufgaben des Historikers. Vortrag gehalten im Historischen Verein zu Münster i. W. am 15. November 1934 (= Philosophie und Geschichte 53). Tübingen: Mohr.

Hasselhorn, Benjamin (Bearb.)/Kleinert, Christian (Vorarb.) (2014): Johannes Haller (1865-1947). Briefe eines Historikers (Deutsche Geschichtsquellen des 19. und 20. Jahrhunderts 71). München: de Gruyter.

Heiber, Helmut (1966): Walter Frank und sein Reichsinstitut für Geschichte des neuen Deutschlands (= Quellen und Darstellungen zur Zeitgeschichte 13). Stuttgart: Deutsche Verlags-Anstalt.

Kraus, Hans-Christof (2011): Über einige geistesgeschichtliche Voraussetzungen des Nationalsozialismus. In: Becker, M./Bongartz, S. (Hrsg.): Die weltanschaulichen Grundlagen des NS-Regimes. Ursprünge, Gegenentwürfe, Nachwirkungen. Tagungsband der XXIII. Königswinter Tagung im Februar 2010 (= Schriftenreihe der Forschungsgemeinschaft 20. Juli e.V. 15). Berlin u.a.: LIT Verlag, S. 21-40.

Meinecke, Friedrich (1946/1969): Die deutsche Katastrophe In: Meinecke, F.: Autobiographische Schriften (Werke, Band 8). Stuttgart: Koehler, S. 323-445.

Nonn, Christoph (2013): Theodor Schieder. Ein bürgerlicher Historiker im 20. Jahrhundert (Schriften des Bundesarchivs 73). Düsseldorf: Droste.

Nonn, Christoph (2014): Erwiderung auf Peter Schöttlers Rezension meiner Biographie über Theodor Schieder. In: H-Soz-u-Kult 09.01.2014. http://h sozkult.geschichte.huberlin.de/rezensionen/type=rezbuecher\&count=1\&r ecno $=1 \&$ sort $=$ datum\&order $=$ down\&id $=21182 \&$ segment_ignore $=224 \&$ re $=2298 \#$ re2298 $>$ [Zugriff: 22. Oktober 2014].

Real, Willy (1997): Zwischen Zuversicht und Entartung. Erinnerungen an ein Studium der Geisteswissenschaften in den Jahren vor und nach der nationalsozialistischen Machtergreifung 1930-1935 (Lebenserinnerungen 3). Hamburg: Kovač.

Reichert, Folker (2009): Gelehrtes Leben. Karl Hampe, das Mittelalter und die Geschichte der Deutschen (= Schriftenreihe der Historischen Kommission bei der Bayerischen Akademie der Wissenschaften 79). Göttingen: Vandenhoeck \& Ruprecht. 
Ritter, Gerhard (1947): Die Dämonie der Macht. Betrachtungen über Geschichte und Wesen des Machtproblems im politischen Denken der Neuzeit. 5., umgearbeitete Auflage des erstmals 1940 erschienenen Buches „Machtstaat und Utopie“. Stuttgart: Hannsmann.

Rothfels, Hans (1965): Die Geschichtswissenschaft in den dreißiger Jahren. In: Flitner, A. (Hrsg.): Deutsches Geistesleben und Nationalsozialismus. Eine Vortragsreihe der Universität Tübingen. Tübingen: Wunderlich, S. 90-107.

Schleier, Hans (1975): Die bürgerliche deutsche Geschichtsschreibung der Weimarer Republik. I. Strömungen - Konzeptionen - Institutionen. II. Die linksliberalen Historiker (= Akademie der Wissenschaften der DDR. Schriften des Zentralinstituts für Geschichte 40). Berlin: Akademie-Verlag.

Schönwälder, Karen (1992): Historiker und Politik. Geschichtswissenschaft im Nationalsozialismus (= Historische Studien 9). Frankfurt am Main u.a.: Campus.

Schöttler, Peter (2007): Deutsche Historiker auf vermintem Terrain. Einleitende Bemerkungen. In: Pfeil, U. (Hrsg.): Das Deutsche Historische Institut in Paris und seine Gründungsväter. Ein personengeschichtlicher Ansatz (Pariser Historische Studien 86). München: Oldenbourg, S. 15-31.

Schöttler, Peter (2013): Nonn, Christoph: Theodor Schieder. Ein bürgerlicher Historiker im 20. Jahrhundert. In: H-Soz-u-Kult 19.12.2013, http://hsozk ult.geschichte.hu-berlin.de/rezensionen/2013-4-227 [Zugriff: 22. Oktober 2014].

Schulze, Winfried (1989) : Deutsche Geschichtswissenschaft nach 1945 (= Historische Zeitschrift, Beiheft 10). München: Oldenbourg.

Schulze, Winfried (1997): Doppelte Entnazifizierung. Geisteswissenschaften nach 1945. In: König, H./ Kuhlmann, W./Schwabe, K. (Hrsg.): Vertuschte Vergangenheit. Der Fall Schwerte und die NS-Vergangenheit der deutschen Hochschulen. München: Beck, S. 257-286.

Schulze, Winfried/Helm, Gerd/Ott, Thomas (1999): Deutsche Historiker im Nationalsozialismus. Beobachtungen und Überlegungen zu einer Debatte. In: Schulze, W./Oexle, O. G: (Hrsg.): Deutsche Historiker im Nationalsozialismus. Frankfurt am Main: Fischer Taschenbuch Verlag, S. 1148.

Thimme, David (2006): Percy Ernst Schramm und das Mittelalter. Wandlungen eines Geschichtsbildes (Schriftenreihe der Historischen Kommission bei der Bayerischen Akademie der Wissenschaften 75). Göttingen: Vandenhoeck \& Ruprecht.

Wehler, Hans-Ulrich (1983): Das deutsche Kaiserreich 1871-1918. Göttingen: Vandenhoeck \& Ruprecht. 
Wehler, Hans-Ulrich (1999): Nationalsozialismus und Historiker. In: Schulze, W./Oexle, O. G. (Hrsg.): Deutsche Historiker im Nationalsozialismus. Frankfurt am Main: Fischer Taschenbuch Verlag, S. 306-339.

Wehler, Hans-Ulrich (2006): Eine lebhafte Kampfsituation. Ein Gespräch mit Manfred Hettling und Cornelius Torp. München: C.H. Beck Verlag.

Werner, Karl Ferdinand (1967): Das NS-Geschichtsbild und die deutsche Geschichtswissenschaft. Stuttgart u.a.: Kohlhammer.

Wolf, Ursula (1996): Litteris et Patriae. Das Janusgesicht der Historie (= Frankfurter Historische Abhandlungen 37). Stuttgart: Franz Steiner Verlag. 